\title{
Quantum ballistic conductance of quasi-two-dimensional and three-dimensional semiconductor nanowires
}

\author{
Luis C. O. Dacal and Álvaro J. Damião \\ Instituto de Estudos-Avançados_IEAv—CTA, C.P. 6044, 12231-970 São José dos Campos—SP, Brazil \\ Erasmo A. de Andrada e Silva \\ Instituto Nacional de Pesquisas Espaciais—INPE, C.P. 515, 12201-970 São José dos Campos—SP, Brazil \\ (Received 16 October 2004; revised manuscript received 7 January 2005; published 29 April 2005)
}

\begin{abstract}
With the solution of the Schrödinger equation for electrons in three-dimensional (3D) hard wall quantum channels, the conductance of semiconductor nanowires is studied as a function of length, size, and contact dimensionality. Within the envelope function approximation, the two-terminal Landauer-Büttiker conductance has been calculated in the quantum ballistic regime, using the mode matching technique. The contacts are modeled by semi-infinite regions with hard wall confinement along only one of the transverse directions, so that continuous crossover from quasi-two-dimensional to 3D contacts can be simulated through the increase of this confinement length. The conductance resonances due to the resonant transmission through quasi-bound longitudinal states are shown to get much better resolved with 3D contacts, which leads to larger Fabry-Pérot like conductance oscillations within the $2 e^{2} / h$ quantized plateaus, which are independent of the contact dimension. An effective phase shift due to electron reflection at the exit and entrance of the quantum channel is introduced, which helps the interpretation of the numerical and experimental data on these conductance oscillations.
\end{abstract}

DOI: 10.1103/PhysRevB.71.155330

PACS number(s): 73.21.Hb, 73.23.Ad, 73.63.Nm

\section{INTRODUCTION}

After the experimental discovery of the conductance quantization in semiconductor point contact structures,,${ }^{1,2}$ its close connection with the quantum Hall effect was soon recognized, except for the much smaller precision and the absence of a magnetic field. In fact, both these effects derive from the equipartition of the current among an integer number of propagating modes. ${ }^{3}$ Since then there has been an intense effort toward a better understanding of the conductance quantization which has been observed in semiconductor nanostructures, ${ }^{4-6}$ metallic systems, ${ }^{7,8}$ and atomic wires. ${ }^{9-11}$ However, large $(\sim 20 \%)$ deviations from the quantized steps continue to be observed and not well understood, and it is sometimes referred to as nonuniversal or anomalous conductance quantization. ${ }^{4,12,13}$

It is known that such deviations can have different origins. Electron scattering (or mode coupling) at the entrance and exit of the constriction (quantum channel, point contact, or nanowire), including the electron transmission by tunneling, ${ }^{14-18}$ structure disorder, ${ }^{14,19,20}$ and nonequilibrium thermal effects ${ }^{12,21}$ are among them. In particular, theoretical simulations based on the solution of the Schrödinger equation for two-dimensional (2D) models have shown that the conductance quantization should be seen only in point contacts or nanowires with nonzero length and that the electron scattering at the two wire ends leads to large conductance oscillations within the so-called $2 e^{2} / h$ plateaus, ${ }^{14-16}$ which are obtained by changing the electron density or the conducting channel width. ${ }^{3}$ Such superimposed oscillations have been observed by different groups. ${ }^{1,4,6}$ They correspond to Fabry-Pérot like oscillations due to resonant electron transmission via quasi-bound (longitudinal) states inside the chan- nel; a quantum interference phenomena which is highly sensitive to the specific geometry of the nanowire and contacts. In fact, the study of these oscillations done so far with 2D models, ${ }^{15,22}$ where the out-of-the-plane dynamics is neglected, is not able to answer how these oscillations depend on the wire cross section and on the dimension of the contacts; three-dimensional (3D) models are necessary for that. It is worth mentioning that $3 \mathrm{D}$ models are also required in view of the fabrication of spintronic ${ }^{23}$ devices based on spinorbit coupling, which couples the three coordinates dynamics. $^{24-26}$

Here we present, a study of the quantum ballistic conductance of 3D semiconductor nanowires and its geometric effects within a simple hard wall quantum channel model. The Schrödinger equation for independent electrons is solved with complete mode matching at the entrance and exit of the nanowire. ${ }^{15,22}$ The contacts are modeled by semi-infinite regions with hard wall confinement only along one of the transverse directions. The continuous crossover from quasi-2D to 3D contacts is simulated through the increase of this confinement length. Within the envelope function approximation, we calculate the two-terminal LandauerBüttiker conductance ${ }^{27,28}$ in the quantum coherent regime. The conductance peaks due to resonant tunneling via longitudinal quasi-bound states are shown to get much better resolved with 3D contacts leading to conductance oscillations with larger amplitudes. It is also shown that these oscillations can be simply described with an effective phase shift due to electron scattering at the wire entrance and exit. This shift corresponds to an effective wire length which depends on the contact dimension and Fermi energy, and gives the wavelength of the charge distribution inside the wire. In Sec. II we present our model calculation and in Sec. III we discuss the 

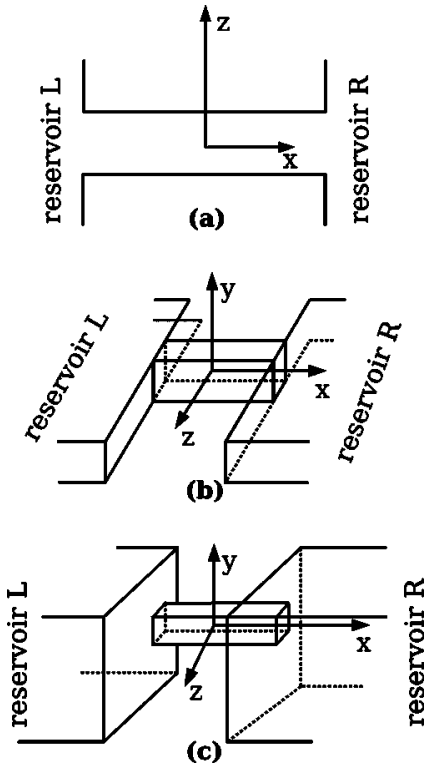

FIG. 1. Schematic representation of 2D (a), quasi-2D (b), and 3D (c) systems. The QWR is the connection between left $(L)$ and right $(R)$ reservoirs, which are infinite in $z$ direction for all systems. The origin of the axes is the center of the QWR.

main results, which are then summarized in the conclusions.

\section{MODEL}

Let us consider a general constriction, quantum channel or nanowire (QWR) between two reservoirs (contacts), all made with hard walls. Figure 1, schematically represents the corresponding $2 \mathrm{D}$, quasi-2D, and $3 \mathrm{D}$ versions of such quantum wire model. It is important to notice that in all cases the reservoirs are infinite in $z$ direction (and semi-infinite in $x$ ). The contact confining length along $y\left(D_{y}\right)$ matches the QWR width $\left(W_{y}\right)$ in the quasi-2D case. On the other hand, in the 3D case, $D_{y}$ is much larger than $W_{y}$. We consider the QWR and contacts made of the same semiconductor material, namely GaAs, which means using electron effective mass $m^{*}=0.067$ in the numerical results. For the $2 \mathrm{D}$ case, simple solutions for the Schrödinger equation using the so-called mode matching technique have been presented by Kirczenow ${ }^{15}$ and Berggren and $\mathrm{Ji}^{22}$ Here, we extend such calculations and present new solutions for the systems represented in Figs. 1(b) and 1(c) with continuous 2D-3D crossover. With the same technique, other 3D structures have been studied by $\mathrm{Xu}^{17}$ and Huaxiang and Xide. ${ }^{18}$

Let us assume an electron incident from the left contact with wave vector $\mathbf{k}=\left(k_{x}, k_{z}\right)$ and occupying the $l$ th subband in $y$ with energy $E_{l}(\mathbf{k})=\hbar^{2} / 2 m^{*}\left\{k_{x}^{2}+k_{z}^{2}+\left(l \pi / D_{y}\right)^{2}\right\}$. We can then write the total wave function in the source reservoir (left contact, $L$ ) as

$$
\Psi^{L}(\mathbf{r})=e^{i k_{x} x+i k_{z} z} \phi_{l}(y)+\sum_{n} \phi_{n}(y) \int_{-\infty}^{+\infty} d k_{z}^{\prime}\left\{A_{n}\left(k_{z}^{\prime}\right) e^{-i k_{x}^{\prime} x+i k_{z}^{\prime} z}\right\},
$$

where $\phi_{n}(y)$ is the eigenfunction for the $n$th subband in the contact and the $A_{n}\left(k_{z}^{\prime}\right)$ are the coefficients of the reflected partial waves due to the electron scattering at the wire entrance. The sum is over all transverse states and, due to energy conservation, the wave vector $k_{x}^{\prime}=\left\{2 m^{*} E_{l} / \hbar^{2}-k_{z}^{\prime 2}\right.$ $\left.-\left(n \pi / D_{y}\right)^{2}\right\}^{1 / 2}$ assumes real and imaginary values, i.e., the evanescent partial waves with $k_{x}^{\prime} / i>0$ are also included. The convention $(-1)^{1 / 2}=+i$ is used throughout the paper.

Similarly, in the drain or right contact $(R)$, the wave function can be written as

$$
\Psi^{R}(\mathbf{r})=\sum_{m} \phi_{m}(y) \int_{-\infty}^{+\infty} d k_{z}^{\prime}\left\{C_{m}\left(k_{z}^{\prime}\right) e^{i k_{x}^{\prime} x+i k_{z}^{\prime} z}\right\}
$$

where evanescent waves are again fully taken into account.

Inside the QWR, the solution is expanded in terms of the QWR eigenfunctions, so that it can be written as

$$
\Psi^{W}(\mathbf{r})=\sum_{n_{y}, n_{z}}\left\{B_{n_{y}, n_{z}}^{+} e^{+i q_{n_{y}, n_{z}} x}+B_{n_{y}, n_{z}}^{-} e^{-i q_{n_{y}, n_{z}} x}\right\} \varphi_{n_{y}}(y) \varphi_{n_{z}}(z),
$$

where $\varphi_{n_{z}}(z)\left(\varphi_{n_{v}}(y)\right)$ represents the $n_{z}^{\text {th }}\left(n_{y}^{\text {th }}\right)$ eigenfunction for the QWR confining potential in $z(y)$ direction. Evanescent, exponentially growing and freely propagating states are considered with $q_{n_{y}, n_{z}}=\left\{2 m^{*} E_{l} / \hbar^{2}-\left(n_{y} \pi / W_{y}\right)^{2}-\left(n_{z} \pi /\right.\right.$ $\left.\left.W_{z}\right)^{2}\right\}^{1 / 2} . L, W_{y}$ and $W_{z}$ are the dimensions of the QWR in $x$, $y, z$ directions, respectively.

In order to obtain the conductance, we calculate the current through the QWR which is given as a function of the coefficients $B_{n_{y}, n_{z}}^{+}$and $B_{n_{y}, n_{z}}^{-}$. These in turn, are determined with the solution of a system of linear equations which results from the matching of the wave functions and their derivatives with respect to $x$ at $x=+L / 2$ and $x=-L / 2$, plus the elimination of the coefficients $A_{n}\left(k_{z}^{\prime}\right)$ and $C_{m}\left(k_{z}^{\prime}\right)$. For the quasi-2D case these equations read:

$$
\begin{aligned}
& \sum_{n_{z}} B_{n_{y}, n_{z}}^{+} e^{-i\left(q_{n_{y}, n_{z}} L / 2\right)}\left(T_{m_{z}, n_{z}}+W_{z} \pi q_{n_{y}, n_{z}} \delta_{n_{z}, m_{z}}\right) \\
&+\sum_{n_{z}} B_{n_{y}, n_{z}}^{-} e^{+i\left(q_{n_{y}, n_{z}} L / 2\right)}\left(T_{m_{z}, n_{z}}-W_{z} \pi q_{n_{y}, n_{z}} \delta_{n_{z}, m_{z}}\right) \\
&=\frac{2 \sqrt{2}}{\sqrt{W_{y}}} k_{x} e^{-i\left(k_{x} L / 2\right)} M_{+k_{z}, m_{z}} \delta_{l, n_{y}}, \\
& \sum_{n_{z}} B_{n_{y}, n_{z}}^{+} e^{+i\left(q_{n_{y}, n_{z}} L / 2\right)}\left(T_{m_{z}, n_{z}}-W_{z} \pi q_{n_{y}, n_{z}} \delta_{n_{z}, m_{z}}\right) \\
&+\sum_{n_{z}} B_{n_{y}, n_{z}}^{-} e^{-i\left(q_{n_{y}, n_{z}} L / 2\right)}\left(T_{m_{z}, n_{z}}+W_{z} \pi q_{n_{y}, n_{z}} \delta_{n_{z}, m_{z}}\right)=0
\end{aligned}
$$

where $\delta_{n_{z}, m_{z}}$ is the usual Kronecker delta and

$$
T_{m_{z}, n_{z}}=\int_{-\infty}^{+\infty} d k_{z}^{*} k_{x}^{*} M_{+k_{z}^{*}, m_{z}} M_{-k_{z}^{*}, n_{z}}
$$




$$
M_{-k_{z}^{*}, n_{z}}=\int_{-W_{z} / 2}^{+W_{z} / 2} d z \varphi_{n_{z}}(z) e^{-i\left(k_{z}^{*} z\right)},
$$

where $k_{x}^{*}=\left\{2 m^{*} E_{l} / \hbar^{2}-k_{z}^{* 2}-\left(n \pi / D_{y}\right)^{2}\right\}^{1 / 2}$ is function of $k_{z}^{*}$ through the energy conservation in the contacts.

Similarly, for the 3D case we have

$$
\begin{aligned}
& \sum_{n_{y}, n_{z}} B_{n_{y}, n_{z}}^{+} e^{-i\left(q_{n_{y}, n_{z}} L / 2\right)}\left(\sum_{l_{y}} M_{l_{y}, m_{y}} M_{l_{y}, n_{y}} T_{m_{z}, n_{z}}\right. \\
& \left.\quad+\frac{W_{y}}{4} W_{z} D_{y} \pi q_{m_{y}, m_{z}} \delta_{m_{y}, n_{y}} \delta_{m_{z}, n_{z}}\right)+\sum_{n_{y}, n_{z}} B_{n_{y}, n_{z}} e^{+i\left(q_{n_{y}, n_{z}} L / 2\right)} \\
& \quad \times\left(\sum_{l_{y}} M_{l_{y}, m_{y}} M_{l_{y}, n_{y}} T_{m_{z}, n_{z}}-\frac{W_{y}}{4} W_{z} D_{y} \pi q_{m_{y}, m_{z}} \delta_{m_{y}, n_{y}} \delta_{m_{z}, n_{z}}\right) \\
& =2 \pi \sqrt{2 D_{y}} k_{x} e^{-i\left(k_{x} L / 2\right)} M_{+k_{z}, m_{z}} M_{l, m_{y}},
\end{aligned}
$$$$
\sum_{n_{y}, n_{z}} B_{n_{y}, n_{z}}^{+} e^{+i\left(q_{n_{y}, n_{z}} L / 2\right)}\left(\sum_{l_{y}} M_{l_{y}, m_{y}} M_{l_{y}, n_{y}} T_{m_{z}, n_{z}}\right.
$$$$
\left.-\frac{W_{y}}{4} W_{z} D_{y} \pi q_{m_{y}, m_{z}} \delta_{m_{y}, n_{y}} \delta_{m_{z}, n_{z}}\right)+\sum_{n_{y}, n_{z}} B_{n_{y}, n_{z}}^{-} e^{-i\left(q_{n_{y}, n_{z}} L / 2\right)}
$$$$
\times\left(\sum_{l_{y}} M_{l_{y}, m_{y}} M_{l_{y}, n_{y}} T_{m_{z}, n_{z}}+\frac{W_{y}}{4} W_{z} D_{y} \pi q_{m_{y}, m_{z}} \delta_{m_{y}, n_{y}} \delta_{m_{z}, n_{z}}\right)
$$$$
=0 \text {, }
$$

with

$$
M_{l_{y}, n_{y}}=\int_{-W_{y} / 2}^{+W_{y} / 2} d y \phi_{l_{y}}^{*}(y) \varphi_{n_{y}}(y),
$$

where $\phi_{l_{y}}(y)\left(\varphi_{n_{y}}(y)\right)$ is the eigenfunction for the $l_{y}^{\text {th }}\left(n_{y}^{\text {th }}\right)$ subband in the contact (QWR).

The equations for the quasi-2D case [Eq. (4)] are much simpler (note the absence of the sum over $n_{y}$ ). This is because the wire and contact $y$-confined modes are the same. As a consequence, with only the first subband occupied, i.e., for the first conductance plateau, the obtained quasi-2D conductance and that in the 2D limit are the same except for the energy shift due to the extra confinement in the quasi-2D case. For higher plateaus, contributions from initial states with different subbands must be added through independent solutions of Eq. (4) for the different possible values of $l$ [see the $\delta_{l, n_{y}}$ term in Eq. (4)]. On the other hand, in the 3D case, the sets of $y$-confined states are different in the wire and contacts, and we have $M_{l_{y}, n_{y}} \neq \delta_{l_{y}, n_{y}}$ [see Eq. (7)]. In this case, the only possible simplification is that $T_{m_{z}, n_{z}}$ and $M_{l_{y}, n_{y}}$ are nonzero only for indexes with the same parity.

Finally, assuming zero temperature and a weak potential difference between the reservoirs, the wire conductance can be calculated by ${ }^{22}$

$$
G\left[\frac{2 e^{2}}{h}\right]=-\left.\frac{m^{*}}{e h} \sum_{l=1}^{l_{\max }} \int_{-\pi / 2}^{+\pi / 2} d \theta j_{l}(\mathbf{k})\right|_{|\mathbf{k}|=k_{F}},
$$

where the sum is over all possible initial subbands, $k_{F}$ is the modulus of the Fermi wave vector, $\mathbf{k}=k_{F}(\cos (\theta), \sin (\theta))$ and

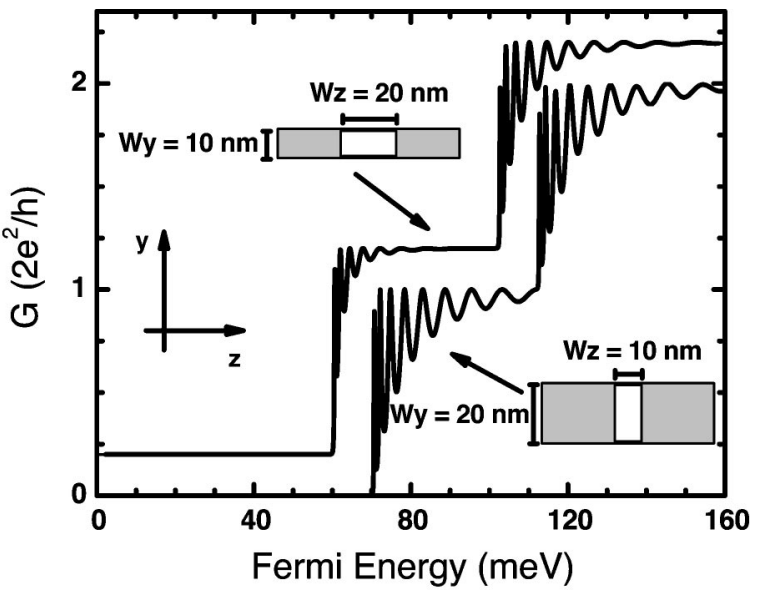

FIG. 2. Conductance in quasi-2D systems as a function of Fermi energy for QWRs with $L=100 \mathrm{~nm}, W_{y}=10 \mathrm{~nm}, W_{z}=20 \mathrm{~nm}$ (upper curve) and $W_{y}=20 \mathrm{~nm}, W_{z}=10 \mathrm{~nm}$ (lower curve). The upper curve is offset for clarity.

the single mode electrical current $j_{l}$ is given by

$$
\begin{aligned}
j_{l}(\mathbf{k})= & -\frac{\hbar e}{m^{*}} \frac{W_{y}}{2} \frac{W_{z}}{2}\left\{\sum_{n_{y}, n_{z}}{ }^{(R)} q_{n_{y}, n_{z}}\left(\left|B_{n_{y}, n_{z}}^{+}\right|^{2}-\left|B_{n_{y}, n_{z}}^{-}\right|^{2}\right)\right. \\
& \left.+\sum_{n_{y}, n_{z}}{ }^{(\mathrm{Im})} q_{n_{y}, n_{z}}\left(B_{n_{y}, n_{z}}^{+} B_{n_{y}, n_{z}}^{-*}-B_{n_{y}, n_{z}}^{+*} B_{n_{y}, n_{z}}^{-}\right)\right\},
\end{aligned}
$$

where $R(\mathrm{Im})$ indicates sum over real (imaginary) values of $q_{n_{y}, n_{z}}$.

In practical calculations, Eqs. (4) and (6) for the coefficients $B_{n_{y}, n_{z}}^{(+,)}$are solved numerically by truncating the number of transversal modes. Fortunately, the contribution of modes with energy above the Fermi level decreases rapidly with the mode index. Good convergence of the results (within 1\%) have been obtained already with only two modes above the Fermi level. In the results shown in the following, this corresponds to the use of up to five modes for the wider dimension. The 3D results are obtained with $D_{y}=200 \mathrm{~nm}$ and 300 $y$-confined levels used to perform the sums over $l_{y}$ in Eq. (6). The obtained solutions of Eq. (6) reproduce exactly previous 3D results, including the double plateaus due to mode degeneracy ${ }^{17}$ and Eq. (4), in the appropriate limit, also reproduce exactly previous results for strictly $2 \mathrm{D}$ channels. ${ }^{15} \mathrm{In}$ Sec. III, we discuss the results for the quasi-2D case and the 2D-3D quantum ballistic conductance crossover, including the discussion of the conductance oscillations in terms of effective wire length.

\section{RESULTS AND DISCUSSIONS}

Figure 2 shows typical results of the quasi-2D model. We have plotted there the obtained conductance as a function of the electron Fermi energy for two $20 \times 10 \mathrm{~nm}^{2}$ rectangular QWRs, $100 \mathrm{~nm}$ long, being one with the longer side parallel to the quasi-2D contact (upper curve) and one perpendicular (lower curve), as illustrated in the figure. It is seen that in the perpendicular case the oscillations are stronger corresponding to better defined transmission resonances. Note that in 


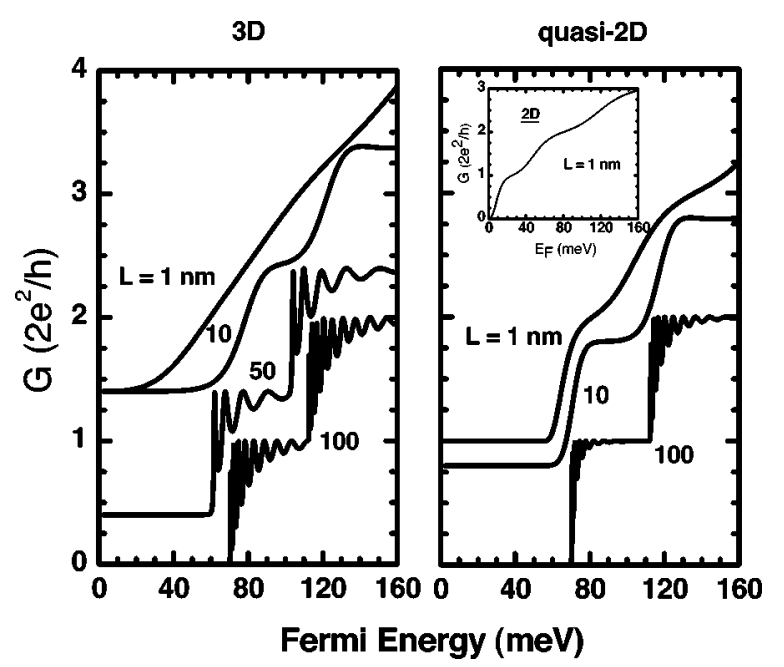

FIG. 3. Obtained conductance with 3D (left panel) and quasi-2D (right panel) contacts as a function of Fermi energy for QWRs with $W_{y}=10 \mathrm{~nm}$ and $W_{z}=20 \mathrm{~nm}$. $L$ varies from 1 to $100 \mathrm{~nm}$ as indicated. Inset: $2 \mathrm{D}$ system results for $L=1 \mathrm{~nm}$. The upper curves are offset for clarity.

the limit $W_{z} \rightarrow \infty$, one cannot distinguish wire and contact, there is no scattering (or reflection) at the entrance and exit of the wire and, as a consequence, no conductance oscillations. As $W_{z}$ is reduced, the electron modes inside and outside the wire become more and more dissimilar, increasing the electron scattering at the entrance and exit of the wire, which leads to better defined longitudinal resonances and larger oscillation amplitudes.

In Fig. 3, we compare the conductance of nanowires with varying length $L$ obtained with the 3D (left panel), quasi-2D (right panel), and 2D (inset) models. The wire length $L$ controls the conductance oscillation frequency; the longer the wire the more longitudinal resonances can be seen by increasing the Fermi energy. In the limit of very short wires, the first oscillation period cannot be completed within the respective plateaus which are no more distinguishable also due to an increasing tunneling contribution to the electron transport. As a result, in this limit, an almost linear behavior is obtained for the conductance in all three models. Electron tunneling also occurs at energies smaller than the QWR fundamental transversal eigenenergy and that is why there is no conductance threshold energy for short QWRs in 3D and 2D cases. In the quasi-2D case instead, there is a threshold energy due to the electron confinement in the contacts. However the main difference between $3 \mathrm{D}$ and quasi-2D results is the much larger oscillation amplitude obtained with 3D contacts. As discussed earlier, this means that with 3D contacts the transmission resonances get better resolved as a result of the smaller electron transparency of the entrance and exit of the QWR. The obtained 2D and quasi-2D results for the first conductance plateau, in all cases considered, turned out to be exactly the same, except for the energy shift, as expected.

In order to further understand these increased conductance oscillations with 3D contacts, in Fig. 4 we have plotted their amplitude (averaged over the first plateau) as a function of $D_{y}$, showing the mentioned quasi-2D to 3D crossover. Three important features should be noted: (i) the fast in-

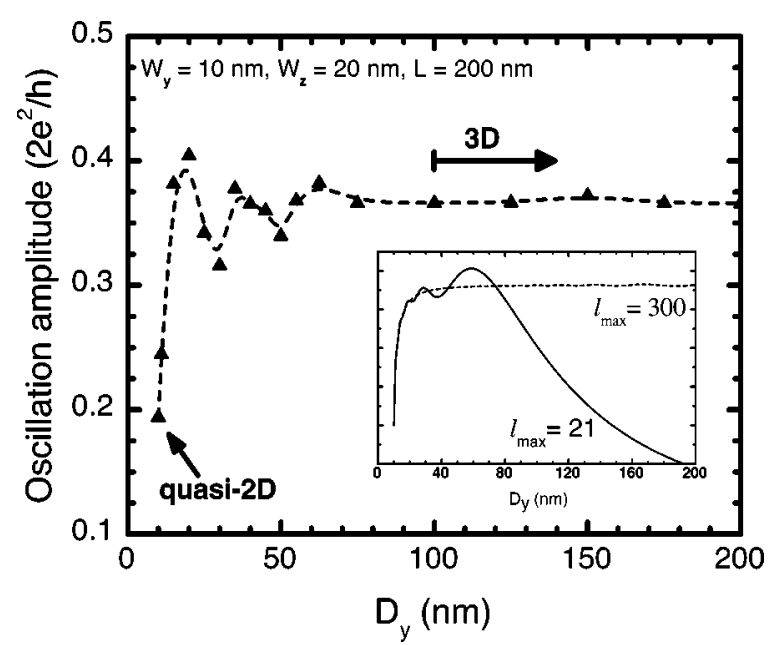

FIG. 4. Mean oscillation amplitude in the first conductance plateau as a function of the contact length $D_{y}$. The QWR dimensions are $L=200 \mathrm{~nm}, W_{y}=10 \mathrm{~nm}$, and $W_{z}=20 \mathrm{~nm}$. The dashed line is just a guide to the eyes. Inset: sum of overlap integrals for the fundamental QWR mode [Eq. (7)] as a function of contact length $D_{y}$. The same QWR dimensions are considered with 21 (full line) and 300 (dashed line) contact modes.

crease when leaving the quasi-2D limit, (ii) the oscillations for small $D_{y}$, and (iii) the saturation in the 3D limit. All these features can be understood by recalling that the conductance oscillations are a result of the electron scattering at the entrance and exit of the QWR, which in turn is determined in a complicated way [see Eqs. (4) and (6)] by the overlap integrals $M_{-k_{z}, n_{z}}$ and $M_{l_{y}, n_{y}}$ [Eqs. (5b) and (7)]. The description of their contribution to the solutions of Eq. (6) is highly nontrivial. Despite this, for the first plateau, the sum of the overlap integrals between the fundamental mode in the QWR and the different modes in the contact $\left(\sum_{l=1}^{l_{\max }} M_{l, 1}\right)$ gives a useful measure of their effects. Indeed, as shown in the inset of Fig. 4, this sum as a function of $D_{y}$ presents the same three features. In the 3D limit, a larger number of contact modes are required and this sum (with $l_{\max }=300$, used in this limit for the required numerical precision), as the amplitude of the conductance oscillations, is seen to saturate. Near the quasi-2D limit, only a small number of contact modes is required and the obtained sum (with $l_{\max }=21$ ) oscillates as the conductance amplitude. The fast increase for small deviations from the quasi-2D limit is also observed. Note that in the quasi-2D case, the $y$-confined modes inside and outside the wire are the same and due to their orthogonality only $M_{1,1}$ is different from zero. With the break of symmetry with $D_{y}>W_{y}$ the observed fast increase in the oscillation amplitude is explained with the contribution of the many different overlap integrals which immediately became different from zero.

As seen, in analogy with the usual parallel mirrors FabryPérot oscillations, the amplitude of the conductance oscillations, corresponding to a series of resonant transmissions, increases with decreasing transparency of the mirrors (i.e., channel entrance and exit) as the resonances become better resolved, with longer lifetimes. Besides for this resonance broadening, the Fabry-Pérot analogy is helpful also in the 


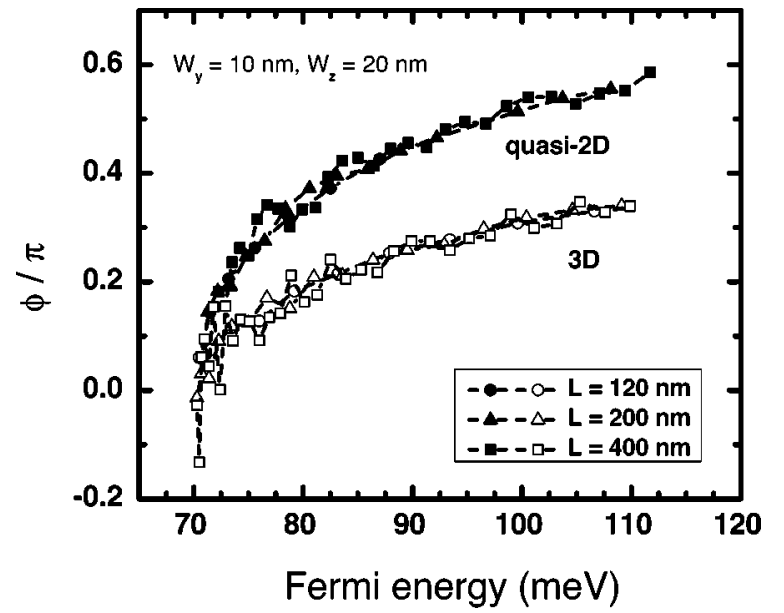

FIG. 5. Effective phase shift for electron reflection at the QWR exit and entrance with 3D (open symbols) and quasi-2D (closed symbols) contacts, as a function of Fermi energy. The QWR dimensions are $W_{y}=10 \mathrm{~nm}, W_{z}=20 \mathrm{~nm}$ and $L=120 \mathrm{~nm}$ (circles), $L$ $=200 \mathrm{~nm}$ (triangles), $L=400 \mathrm{~nm}$ (squares). The lines are just guides to the eyes.

description of the energy positions of these resonances, which can be determined by simple phase relations for the constructive interference between multiple reflected waves. Despite the fact that the electron wave functions in 3D quantum channels have many different components due to the lateral confinement (one from each quantized subband), one can introduce a single effective phase-shift $\phi$ due to reflection at the exit and entrance of the channel so that the usual constructive interference condition will read $\left(2 \pi / \lambda_{F}\right) L+\phi$ $=n \pi$, where $\lambda_{F}$ is the de Broglie wavelength of the Fermi electrons at resonance and $n$ is the corresponding resonance index. In Fig. 5, this effective phase shift for the resonances in the first plateau and different channels is plotted. As $a$ posteriori validation of the analogy, it is seen to be small (with respect to $\pi$ ) and independent of the channel length $L$. Compared to 3D contacts, quasi-2D ones are seen to lead to effective phase shifts twice as big. For the experimental data, this phase shift can be easily obtained to give a measure of the contact dimension.

There is a very simple physical picture for this phase shift. By setting $\phi=\left(2 \pi / \lambda_{F}\right) \delta L$, we can alternatively use the simpler resonance condition $\left(2 \pi / \lambda_{F}\right) L_{\text {eff }}=n \pi$ with an effective wire length $L_{\text {eff }}=L+\delta L$; which gives the wavelength of the resonant states inside the wire. As shown in Fig. $6, \delta L / L$ decreases with increasing wire length and, except near the plateau edge, where it oscillates, it is nearly independent of the Fermi energy. It is of the order of $10^{-2}$ as in the $2 \mathrm{D}$ case $^{15}$ and, for the same wire, $\delta L / L$ with quasi-2D contacts is sensibly larger than with $3 \mathrm{D}$ ones. This can be viewed as due to the extra confinement in the quasi-2D contacts which favors a longer wave-function penetration of the quasi-bound longitudinal states into the contacts along the wire axis. To better see it, in Fig. 7, we have plotted, for example, the resulting density of probability inside the wire $(L=120 \mathrm{~nm})$ along its axis, for the resonances $n=2$ and $n=6$ in the two cases, i.e., with $3 \mathrm{D}$ and quasi-2D contacts, which clearly supports

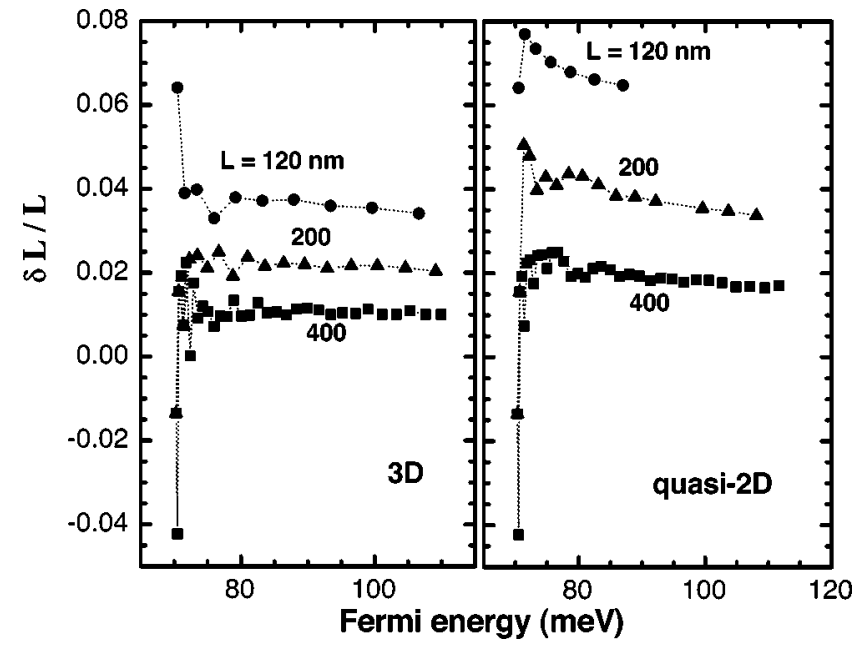

FIG. 6. Effective phase shift in terms of $(\delta L / L)$ for the cases with 3D (left panel) and quasi-2D (right panel) contacts, as a function of Fermi energy for QWRs with $W_{y}=10 \mathrm{~nm}, W_{z}=20 \mathrm{~nm}$ and $L=120 \mathrm{~nm}$ (circles), $L=200 \mathrm{~nm}$ (triangles), $L=400 \mathrm{~nm}$ (squares).

the present simple picture for the conductance resonances. The $n=2$ resonance is seen to correspond in fact to $\lambda=L_{\text {eff }}$ (note that, in the figure, we plot $|\psi|^{2}$, not $\psi$ ), and as expected in accord with Fig. 6, we get $\lambda_{q 2 \mathrm{D}}>\lambda_{3 \mathrm{D}}>L$. Similarly, for $n=6$ we have $3 \lambda=L_{\text {eff }}$.

\section{SUMMARY AND CONCLUSIONS}

We have studied the quantum ballistic conductance of semiconductor QWRs and their geometric effects with a comparative analysis of $2 \mathrm{D}$, quasi-2D and $3 \mathrm{D}$ hard wall models. We have employed the mode matching technique and Landauer-Büttiker formalism to calculate the quantum

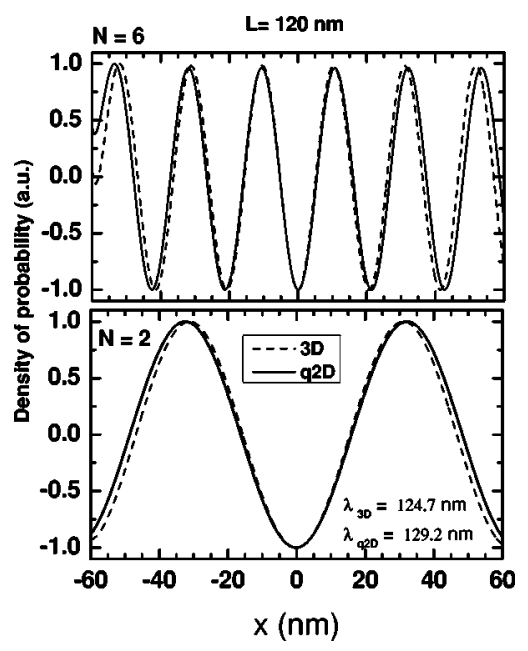

FIG. 7. Density of probability inside the wire (along its axis) at two different resonances, sixth (upper panel) and second (lower panel), for a QWR with $L=120 \mathrm{~nm}, W_{y}=10 \mathrm{~nm}$, and $W_{z}=20 \mathrm{~nm}$. Results with both 3D (dashed line) and quasi-2D (full line) contacts are shown. In the lower panel, the respective effective wavelengths are indicated. Different arbitrary units are used to better compare the resulting wavelengths. 
ballistic conductance in the linear response regime. The conductance oscillations due to resonant transmissions for the same nanowire are shown for example to present with 3D contacts amplitudes much larger than with quasi-2D ones. The continuous quasi-2D to $3 \mathrm{D}$ contact crossover in the quantum conductance of nanowires is obtained and explained in terms of the different electron scattering at the nanowire entrance and exit. An effective phase shift in the electron wave function due to this scattering, with a very simple physical picture, is introduced and shown to be particularly helpful in the analysis and interpretation of both numerical and experimental data. The present results on the geometrical aspects of the quantum ballistic conductance of 3D nanowires should be checked experimentally and can help the characterization and fabrication of actual nanoelectronic devices.

\section{ACKNOWLEDGMENTS}

This work was supported by FAPESP and CNPq (Brazil). Discussions with Professor G. C. La Rocca are also acknowledged.
${ }^{1}$ B. J. van Wees, H. van Houten, C. W. J. Beenakker, J. G. Williamson, L. P. Kouwenhoven, D. van der Marel, and C. T. Foxon, Phys. Rev. Lett. 60, 848 (1988).

${ }^{2}$ D. A. Wharam, T. J. Thornton, R. Newbury, M. Pepper, H. Ahmed, J. E. F. Frost, D. G. Hasko, D. C. Peacock, D. A. Ritchie, and G. A. C. Jones, J. Phys. C 21, L209 (1988).

${ }^{3}$ C. W. J. Beenakker and H. van Houten, Solid State Phys. 44, 1 (1991).

${ }^{4}$ A. Yacoby, H. L. Stormer, N. S. Wingreen, L. N. Pfeiffer, K. W. Baldwin, and K. W. West, Phys. Rev. Lett. 77, 4612 (1996).

${ }^{5}$ D. Kaufman, Y. Berk, B. Dwir, A. Rudra, A. Palevski, and E. Kapon, Phys. Rev. B 59, R10433 (1999).

${ }^{6}$ P. Debray, O. E. Raichev, P. Vasilopoulos, M. Rahman, R. Perrin, and W. C. Mitchell, Phys. Rev. B 61, 10950 (2000).

${ }^{7}$ C. Shu, C. Z. Li, H. X. He, A. Bogozi, J. S. Bunch, and N. J. Tao, Phys. Rev. Lett. 84, 5196 (2000).

${ }^{8}$ A. E. Meyerovich and I. V. Ponomarev, Phys. Rev. B 67, 165411 (2003).

${ }^{9}$ M. Brandbyge, J.-L. Mozos, P. Ordejón, J. Taylor, and K. Stokbro, Phys. Rev. B 65, 165401 (2002).

${ }^{10}$ S. Okano, K. Shiraishi, and A. Oshiyama, Phys. Rev. B 69, 045401 (2004).

${ }^{11}$ M. Brandbyge, J. Schiøtz, M. R. Sørensen, P. Stoltze, K. W. Jacobsen, J. K. Nørskov, L. Olesen, E. Laegsgaard, I. Stensgaard, and F. Besenbacher, Phys. Rev. B 52, 8499 (1995).

${ }^{12}$ K. Houshangpour and K. Maschke, Phys. Rev. B 59, 12248 (1999).

${ }^{13}$ A. Y. Alekseev and V. V. Cheianov, Phys. Rev. B 57, R6834 (1998).

${ }^{14}$ A. Szafer and A. D. Stone, Phys. Rev. Lett. 62, 300 (1989).

${ }^{15}$ G. Kirczenow, Phys. Rev. B 39, 10452 (1989).

${ }^{16}$ E. Tekman and S. Ciraci, Phys. Rev. B 43, 7145 (1991).

${ }^{17}$ H. Xu, Phys. Rev. B 48, 8878 (1993).

${ }^{18}$ F. Huaxiang and X. Xide, Phys. Rev. B 50, 15009 (1994).

${ }^{19}$ S. He and S. D. Sarma, Phys. Rev. B 48, 4629 (1993).

${ }^{20}$ D. Csontos and H. Q. Xu, Appl. Phys. Lett. 77, 2364 (2000).

${ }^{21}$ A. Alekseev and V. V. Cheianov, Phys. Rev. B 57, R6834 (1998).

${ }^{22}$ K.-F. Berggren and Z. li Ji, Phys. Rev. B 43, 4760 (1991).

${ }^{23}$ I. Žutić, J. Fabian, and S. D. Sarma, Rev. Mod. Phys. 76, 323 (2004).

${ }^{24}$ S. Datta and B. Das, Appl. Phys. Lett. 56, 665 (1990).

${ }^{25}$ E. A. de Andrada e Silva, G. C. L. Rocca, and F. Bassani, Phys. Rev. B 50, 8523 (1994).

${ }^{26}$ E. A. de Andrada e Silva and G. C. L. Rocca, Phys. Rev. B 67, 165318 (2003).

${ }^{27}$ R. Landauer, IBM J. Res. Dev. 1, 223 (1957).

${ }^{28}$ M. Buttiker, Phys. Rev. Lett. 57, 1761 (1986). 